\title{
GENETIC HETEROGENEITY OF DOMINANTLY INHERITED OLIVOPONTOCEREBELLAR ATROPHY (OPCA) IN THE JAPANESE: LINKAGE STUDY OF TWO PEDIGREES AND EVIDENCE FOR THE DISEASE LOCUS ON CHROMOSOME 12q (SCA2)
}

\author{
Tatsuo Ihara, ${ }^{1}$ Hidenao Sasaki, ${ }^{1}, *$ Akemi Wakisaka, ${ }^{2}$ \\ Akio Takada, ${ }^{2}$ Takashi Yoshiki, ${ }^{2}$ Tohru Matsuura, ${ }^{1}$ \\ Takeshi Hamada, ${ }^{3}$ Yoshihiro SuzuKI, ${ }^{4}$ and Kunio TASHIRo ${ }^{1}$ \\ ${ }^{1}$ Department of Neurology and ${ }^{2}$ First Department of Pathology, School of Medicine, \\ Hokkaido University, Kita-ku, Sapporo 060, Japan \\ ${ }^{3}$ Hokuyukai Neurological Hospital, Sapporo 060, Japan \\ ${ }^{4}$ Third Department of Internal Medicine, School of Medicine, Yamagata University, \\ Yamagata 990-23, Japan
}

\begin{abstract}
Summary We did a linkage study of 2 multigenerational pedigrees with dominant olivopontocerebellar atrophy (OPCA) other than SCA1, with chromosome $12 \mathrm{q}$ microsatellites. Multipoint linkage analysis led to the conclusion that the disease locus locates within the $6.2 \mathrm{cM}$ interval between IGF1 and D12S84/D12S105. This result coincides with that of Cuban ataxia pedigrees designated as SCA2. Our study provides genetic evidence that dominant OPCA in the Japanese consists of at least two genetically different disorders; SCA1 and SCA2.
\end{abstract}

Key Words spinocerebellar ataxia, hereditary olivopontocerebellar atrophy, genetic heterogeneity, SCA2, linkage analysis

\section{INTRODUCTION}

Dominantly inherited olivopontocerebellar atrophy (OPCA) is a type of spinocerebellar ataxia, defined clinico-pathologically. Generally, symptoms become evident during adulthood and the course is progressive with times, with ultimately disability. Neuropathologically, cerebellum, pontine nuclei, inferior olivary nucleus, and other areas of the central nervous system are selectively involved. On the basis of linkage studies two type of OPCA were seen to be caused by the gene present on different locus: SCA1 on chromosome 6p22-p23 (Zoghbi et al., 1991; Ranum

Received March 7, 1994; Revised version accepted May 20, 1994.

*To whom correspondence should be addressed. 
et al., 1991) and SCA2 on chromosome 12q23-q24.1 (Gispert et al., 1993). An abnormal expansion of CAG trinucleotide repeat was noted for SCA1 (Orr et al., 1993) and no specific gene defect has heretofore been found in SCA2.

Our previous linkage studies revealed that dominant OPCA in the Japanese is genetically heterogeneous (Sasaki et al., 1992), because not all pedigrees with OPCA showed linkage with D6S89, a flanking marker for SCA1 (Ranum et al., 1991; Zoghbi et al., 1991). Based on this background, we did linkage studies of two Japanese pedigrees with OPCA other than SCA1, to examine the relationship with SCA2.

\section{MATERIALS}

Pedigrees. Two pedigrees (P2 and P35) were selected from those not linked with D6S89. In each pedigree, affected individuals were continuously distributed over 4 or more successive generations, in both males and females. The general pedigree information and clinical features of 8 patients (5 from P2 and 3 from P35) were reported previously (Sasaki et al., 1991). In brief, the two pedigrees share common clinical features characterized by cerebellar ataxia, show eye movement, diminished limb tendon reffexes, positive Babinski reflexes, and decreased vibratory sensation in the lower extremities. Muscle atrophy, coarse axial tremor, or choreiform movements were manifested in pe digree P2, at the terminal stage. Nystagmus, dystonia, limb spasticity, external ophthalmoparesis were infrequent in these patients. Therefore, our pedigrees do not satisfy the clinical criteria of Machado-Joseph disease (Lima and Coutinho, 1980). Brain X-ray CT or MRI study showed atrophy of cerebellum and brain stem. In one patient of $\mathrm{P} 2$, the diagnosis was confirmed neuropathologically at autopsy.

Subjects. Twenty-seven individuals from P2, and 14 from P35 were the subjects for linkage analysis, 10 individuals in P2 and 6 in P35 were affected. Of these 16 patients, 8 were examined by our groups, 5 by other investigators, and the remaining 3 refused neurological tests. The structure of these two pedigrees is presented in Fig. 1 together with the age at onset, and the genotype of the markers presented below.

Age at onset. Age at onset was provided by 8 patients in $\mathrm{P} 2$ and 5 patients in $\mathrm{P} 35$; the mean age at onset was $34.2 \pm 11.4$ years (S.D.), the range being from 17 to 52 years. Of these patients, there were 4 parent-offspring pairs; in these pairs, the age of onset in the offspring was 19 to 27 years younger than in the parents $(21.3 \pm 3.9, n=4)$. The disease manifested at a much younger age in case of transmission from the father $(27.4 \pm 8.1, \mathrm{n}=8)$ than from the mother $(43.3 \pm 5.0, n=4)$.

\section{METHODS}

Microsatellites. From polymorphic DNA markers around the SCA2 locus, 
6 microsatellites were selected for analysis. Information of the primer sequences was obtained from either published reports (Polymeropoulos et al., 1990; Wissenbach et al., 1992) or the Genome Data Base (GDB) at Johns Hopkins University. These oligonucleotide primers were synthesized using a DNA synthesizer (Model 380B, Applied Biosystems).

DNA genotyping. With informed consent, $20 \mathrm{ml}$ of peripheral blood were collected from each subject. Genomic DNA was extracted from peripheral blood or lymphoblastoid cell lines. Polymerase chain reactions (PCR) were performed in a total volume of $6 \mu \mathrm{l}$ containing $50 \mathrm{ng}$ of genomic DNA, 0.4 pmol of each primer, $250 \mu \mathrm{M}$ dNTPs, $50 \mathrm{~mm} \mathrm{KCl}, 10 \mathrm{~mm}$ Tris- $\mathrm{HCl} \mathrm{pH} 8.0,1.5 \mathrm{mM} \mathrm{MgCl}_{2}, 0.01 \%$ gelatin, and $0.5 \mathrm{U}$ of Taq polymerase (Amersham). In each case, the forward primer was end-labeled with ${ }^{32} \mathrm{P}-\boldsymbol{\gamma}$-ATP, using T4 polynucleotide kinase. Samples were processed through 30 cycles of denaturation $\left(94^{\circ} \mathrm{C}\right.$ for $60 \mathrm{~s}$ ), annealing for $60 \mathrm{~s}$, and elongation $\left(72^{\circ} \mathrm{C}\right.$ for $90 \mathrm{~s}$ ), followed by a final extension of $7 \mathrm{~min}$ at $72^{\circ} \mathrm{C}$. Annealing temperature was set at $56^{\circ} \mathrm{C}$ in $\mathrm{D} 12 \mathrm{~S} 76$, and at $55^{\circ} \mathrm{C}$ in others. The PCR products were mixed with $45 \mu \mathrm{l}$ of loading buffer $(95 \%$ formamide, $20 \mathrm{~mm}$ EDTA, $50 \mathrm{mg} / \mathrm{ml}$ of bromophenol blue, and $50 \mathrm{mg} / \mathrm{ml}$ of xylene cyanol), denatured, and then cooled in iced water. Five microliters of the mixture was electrophoresed in 6 or $8 \%$ polyacrylamide sequencing gel containing $7 \mathrm{M}$ urea, at $1,600 \mathrm{~V}$ in $1 \times \mathrm{TBE}$ buffer for 3 to $4 \mathrm{hr}$. The products were visualized by autoradiography at room temperature.

Linkage analysis. Pairwise linkage analyses were made with the LIPED program (Ott, 1974) to determine the probability of linkage between each marker and the disease locus. The gene frequency of the disease was assumed to be $1 / 10,000$ and male and female recombination fraction $(\Theta)$ were regarded as equal. The lod scores were corrected, in an age-dependent manner, with final penetrance of $100 \%$. Genetic distance between the marker loci was calculated, based on the combined results of 4 additional Japanese pedigrees by ILINK in the LINKAGE program package (Lathrop et al., 1984). Three-point analysis was made using the subprogram of LINKMAP of the LINKAGE.

\section{RESULTS}

Pairwise linkage analyses (Table 1)

For all 6 markers, both pedigrees did not reach a significant level of linkage with the disease locus, individually. When the two pedigrees were combined, a significant lod score of 3.468 was obtained for D12S 84 at $\Theta_{\max }=0.04$. D12S105 also showed a significant score of 3.18 at $\Theta_{\max }=0.04$. Other markers showed positive scores at various recombination rates, however, none was significant.

Locus order (Table 2)

According to the CEPH map, the locus order of markers is cen-D12S78- 
Table 1. Pairwise lod scores for the disease locus.

\begin{tabular}{|c|c|c|c|c|c|c|c|c|c|}
\hline \multirow{2}{*}{ Locus } & \multirow{2}{*}{ Pedigree } & \multicolumn{6}{|c|}{ Recombination fraction $(\Theta)$} & \multirow{2}{*}{$Z_{\max }$} & \multirow{2}{*}{$\Theta_{\max }$} \\
\hline & & 0.000 & 0.050 & 0.100 & 0.150 & 0.200 & 0.250 & & \\
\hline \multirow[t]{3}{*}{ D12S78 } & P2 & -5.034 & 1.290 & 1. 348 & 1.263 & 1. 112 & 0.923 & & \\
\hline & P35 & -4.649 & 0.249 & 0.391 & 0.404 & 0.364 & 0.297 & & \\
\hline & Total & -9.683 & 1.539 & 1.739 & 1.667 & 1.476 & 1.220 & 0.100 & 1.739 \\
\hline \multirow[t]{3}{*}{ IGF1 } & P2 & 2.961 & 2.706 & 2.426 & 2.125 & 1.806 & 1.473 & & \\
\hline & P35 & -4.360 & -0.140 & 0.045 & 0.105 & 0.115 & 0.101 & & \\
\hline & Total & -1.469 & 2. 566 & 2.471 & 2.230 & 1.921 & 1.574 & 0.09 & 2. 568 \\
\hline \multirow[t]{3}{*}{ D12S105 } & $\mathbf{P} 2$ & -4.267 & 1.684 & 1.658 & 1.493 & 1.270 & 1.020 & & \\
\hline & P35 & 1.672 & 1.495 & 1.311 & 1. 121 & 0.926 & 0.726 & & \\
\hline & Total & -2.595 & 3. 179 & 2.969 & 2. 614 & 2.196 & 1.746 & 0.04 & 3. 182 \\
\hline \multirow[t]{3}{*}{ D12S84 } & $\mathrm{P} 2$ & -3.583 & 1.924 & 1.903 & 1.738 & 1.504 & 1.228 & & \\
\hline & P35 & 1.723 & 1.542 & 1.354 & 1.160 & 0.959 & 0.754 & & \\
\hline & Total & -1.860 & 3.466 & 3.258 & 2.898 & 2.463 & 1.982 & 0.04 & 3.468 \\
\hline \multirow[t]{3}{*}{ PLA2 } & P2 & -5.692 & -2.108 & -1.104 & -0.582 & -0.268 & -0.077 & & \\
\hline & P35 & 0.760 & 0.657 & 0.552 & 0.447 & 0.342 & 0.244 & & \\
\hline & Total & -4.932 & -1.451 & -0.552 & -0.135 & 0.074 & 0.166 & 0.30 & 0.186 \\
\hline \multirow[t]{3}{*}{ D12S76 } & P2 & -7.449 & -0.785 & -0.097 & 0.202 & 0.335 & 0.374 & & \\
\hline & P35 & 1. 162 & 1.052 & 0.930 & 0.796 & 0.654 & 0.506 & & \\
\hline & Total & -6.288 & 0.268 & 0.833 & 0.998 & 0.989 & 0.880 & 0.17 & 1.110 \\
\hline
\end{tabular}

Table 2. Genetic distance between the $12 \mathrm{q}$ markers.

\begin{tabular}{lccccc}
\hline & Locus combination & $Z_{\max }$ & $\Theta_{\max }$ & $\begin{array}{c}\text { Map distancea } \\
\text { (cM) }\end{array}$ \\
\hline D12S78 & vs & IGF1 & 14.87 & 0.022 & 2.2 \\
IGF1 & vs & D12S105/D12S84 & 13.26 & 0.058 & 6.2 \\
D12S105 & vs & D12S84 & 10.90 & 0.000 & 0.0 \\
D12S105/D12S84 & vs & PLA2/D12S76 & 12.52 & 0.060 & 6.4 \\
PLA2 & vs & D12S76 & 9.59 & 0.000 & 0.0 \\
\hline
\end{tabular}

Distance was calculated from Haldane's map function. 
(D12S105/S84)-D12S79-D12S86-D12S76-tel (Weissenbach et al., 1992). Although IGF1 and PLA2 have also been assigned in this region on chromosome 12q (NIH/CEPH collaborative mapping group, 1992), their exact locations on the CEPH map are not yet determined. Pulst et al. (1993) placed the IGF1 centromeric to D12S105/S84, and PLA2 between D12S79 and D12S86. To determine the location of IGF1 and the genetic distance between adjacent markers, we did a linkage analysis, based on our own data of 6 pedigrees, including P2 and P35 (Table 2). The results indicated that IGF1 locates at $6.2 \mathrm{cM}$ from D12S105/S84 between D12S78 and D12S105/S84. We found no recombinants between PLA2 and D12S76. Therefore, the predicted map order is cen-D12S78-(2.4 cM)-IGF1(6.2 cM)-D12S105/D12S84-(6.4 cM)-PLA2-(0.0 cM)-D12S76-tel.

\section{Haplotype segregation analysis (Fig. 1)}

A haplotype segregation analysis was done to search for an informative recombination site. For convenience, alleles at each locus are numbered depending on their product size. In pedigree P2 (Fig. 1a), a single haplotype of "8-6-5-2-5-1" at lineary ordered markers from D12S78 to D12S76 was segregated in 7 of the 10 affected individuals in 3rd and 4th generation. Two recombinant events were found between D12S105/D12S84 and PLA2 (on individual 4.15 and 4.26), thereby indicating that the disease gene locates centromeric to PLA2. One patient (4.54) shared only the allele " 6 " at IGF1 with other affected individuals. Since all the members of the first and second generation, including her father (individual 3.16) had already died, the recombination site on the affected chromosome could not be determined. One likely possibility is that individual 3.16 carried the disease gene on a recombinant haplotype between IGF1 and D12S105/D12S84, and an additional recombination occurred between D12S78 and IGF1, through transmission from 3.16 to individual 4.54. In pedigree P35 (Fig. 1b), a specific haplotype of " $8-2$ $5-12-7-6$ " was segregated in 5 of 6 affected individuals. There was one recombinant event in an unaffected individual (3.04); he died of cardiac disease at age 42 , within at-risk age. Thus, reliable information to localize the disease gene was not obtained. Another recombinant event between IGF1 and D12S105/D12S84 occurred for individual 3.02, thereby indicating that the disease gene locates telomeric to IGF1. These three informative recombinant events from two pedigrees place the disease locus between IGF1 and D12S105/D12S84. Furthermore, since 3 individuals in at-risk generations (5.26 and 6.08 in P2, and 4.01 in P35) inherited the disease gene from their affected parents, they were likely to be potential carriers.

\section{Multipoint linkage analysis (Fig. 2)}

On the basis of the locus order and calculated genetic distances between adjacent markers, multipoint analysis for the disease locus was made. D12S105 and D12S84, and D12S76 and PLA2 were regarded as a single locus, respectively, because no recombination was observed. In the intervals tested, the highest lod 


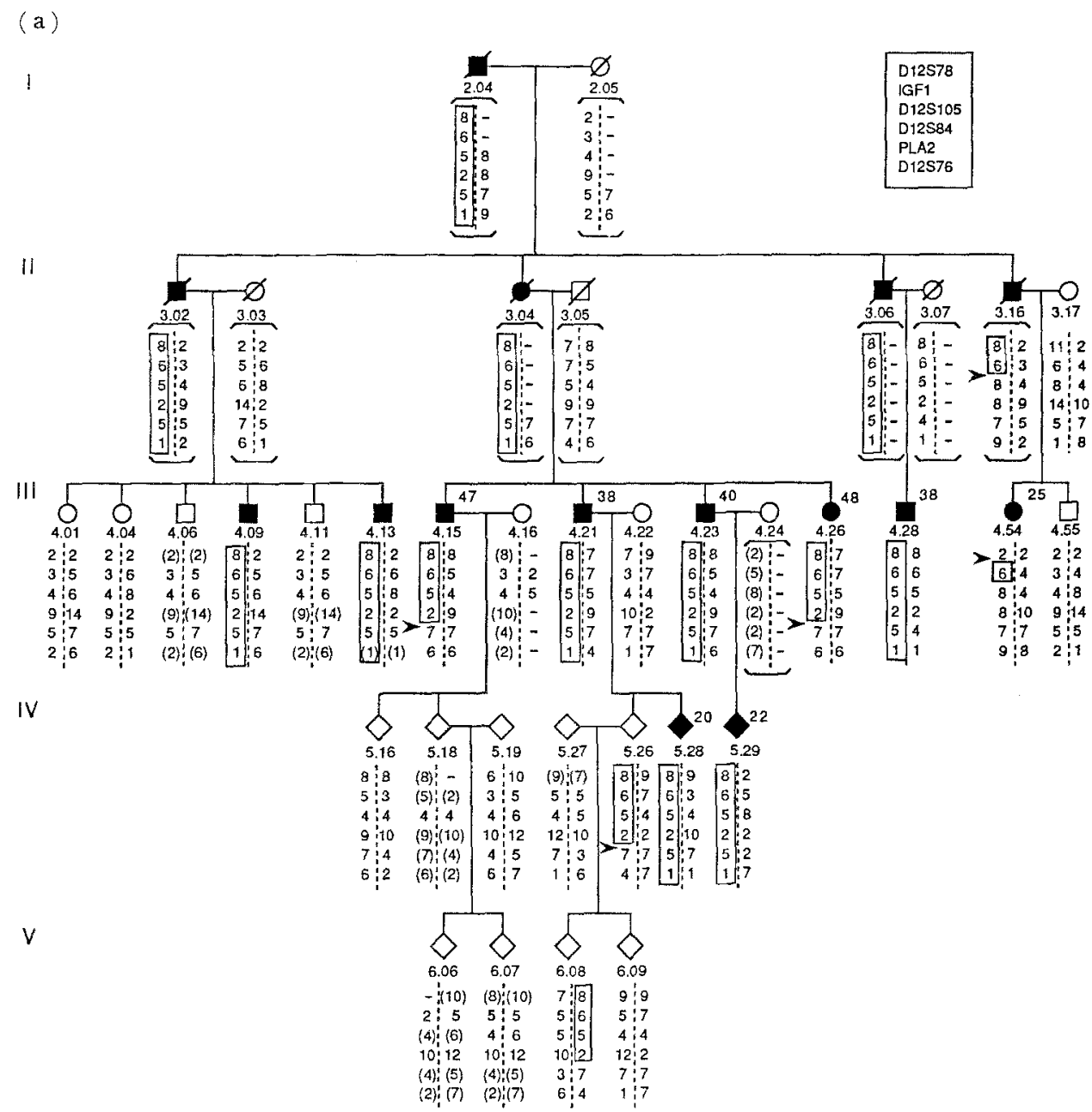

Fig. 1. Pedigree structure and haplotypes of chromosome 12q markers. Deduced haplotypes of deceased individuals are bracketed. Genotypes are in parentheses or dashed, when the phases could or could not be determined, respectively. (a) pedigree P2, (b) pedigree P35. $\square \mathbf{\square}$, male; $\bigcirc \bullet$, female; $\diamond \bullet$, at risk generation; $\bullet \bullet$, affected. Age at onset were presented over the symbol and arrowheads indicate the recombination sites.

score was obtained between IGF1 and D12S105/S84. The maximum score $\left(Z_{\max }\right)$ was 5.0 at $3.2 \mathrm{cM}$ centromeric position from D12S105/S84. The lod score of next probable interval of D12S78-IGF1 is 1.3 at $1 \mathrm{cM}$ centromeric side from IGF1. The peak lod scores from these two intervals were significantly different, hence the disease gene may locate at the IGF1-D12S105/S84 interval 5,404 times more likely than the probable interval of D12S78-IGF1. 
(b)

i

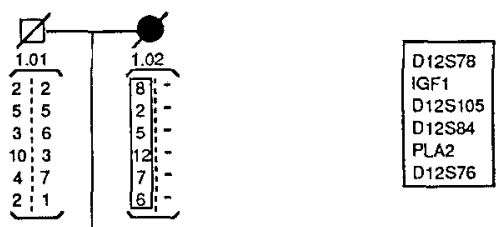

II

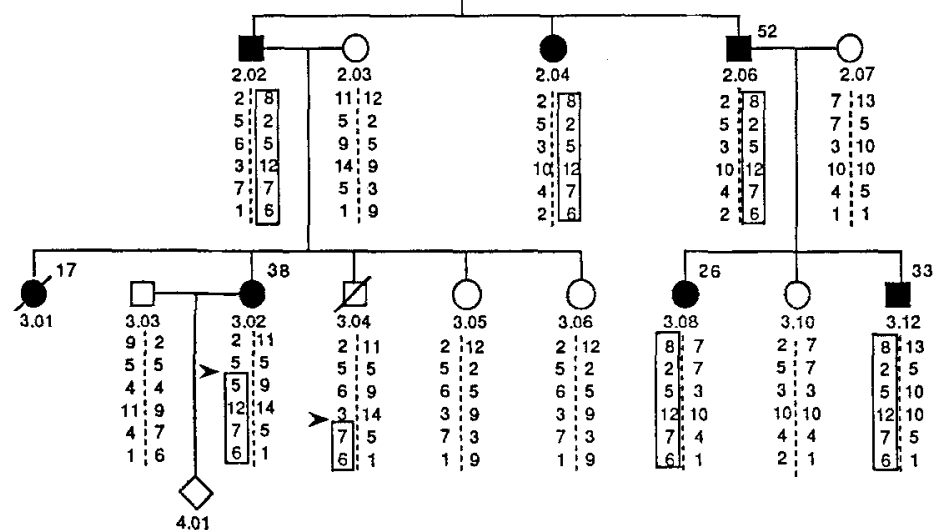

IV

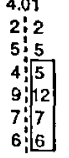

Fig. 1.

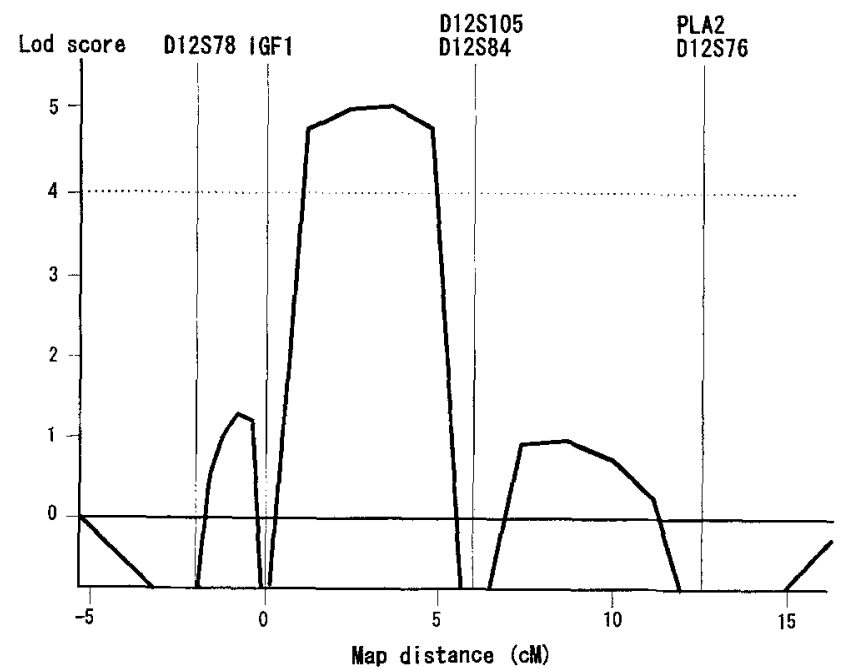

Fig. 2. Three-point lod score curve for the disease locus. 


\section{DISCUSSION}

The locus of SCA2 was first determined by linkage study with pedigrees of Spanish descendants in Cuba (Gispert et al., 1993). The disease locus was mapped within the interval between PLA2 and D12S58 on chromosome 12q23-q24.1. Pulst et al. (1993) then studied another SCA2 pedigree of Italian descendants in the U.S.A., and narrowed the candidate region to a $8.9 \mathrm{cM}$ interval flanked by IGF1 and D12S105/S84. In the present study, the two markers of D12S105 and D12S84 also showed significant linkage with the disease locus. We could not prove a linkage with IGF1, but the multi-locus analysis revealed that the most probable location of the disease locus was on the interval flanked by IGF1 and D12S105/ D12S84.

It is difficult to ascertain the relationship between the present OPCA found in the Japanese and SCA2 in others who are living in geographically separate areas. The close linkage, however, with the same genetic markers as SCA2 strongly suggests that the present OPCA belongs to SCA2 and may possibly be caused by a related genetic defect, although a possible allelic heterogeneity would need to be researched. The molecular genetic background of the Japanese dominant OPCA has not been fully investigated. Among 15 pedigrees, we found that only $55 \%$ were linked with D6S89, a flanking marker of SCA1 (Sasaki et al., 1992). Subsequent analyses of the CAG trinucleotide repeat in the SCAl gene confirmed this heterogeneity (Suzuki et al., manuscript in preparation). Our study provides genetic evidence that $\mathrm{SCA} 2$ is present in the Japanese and the dominant OPCA in the Japanese likely includes at least two genetically different disorders; SCA1 and SCA2.

An abnormally expanded CAG trinucleotide repeat which is characteristic to SCA1 was not detected in the present pedigrees (unpublished data), however, the age at onset is earlier with each generation (anticipation phenomenon). This tendency is particularly evident in case of in paternal transmission. Statistically, we could not prove the difference in intergenerational changes between paternal and maternal transmission, because of limited information on maternal transmission. The "anticipation phenomenon," however, was observed in the SCA2 pedigree (Pulst et al., 1993), and in SCA1 (Chung et al., 1993). Although it is uncertain whether SCA2 is caused by an unstable trinucleotide expansion, as is the case in the other disorders related to genetic anticipation, our observations do suggest that a similar molecular mechanism underlies this disorder.

Acknowledgments We are grateful to the family members for their participation in this study and thank Kunihiko Masuda for technical assistance and Mariko Ohara for reading the manuscript. This study was supported in part by a Research Grant for Intractable Disease from the Ministry of Health and Weifare of Japan, Grant-in-Aid for Scientific Research on Priority Areas, Ministry of Education, Science and Culture of Japan and Suhara Memorial Foundation. 


\section{REFERENCES}

Chung M, Ranum LPW, Duvick LA, Servadio A, Zoghbi HY, Orr HT (1993): Evidence for a mechanism predisposing to intergenerational $C A G$ repeat instability in spinocerebellar ataxia type 1. Nature Genet 5: 254-258

Gispert S, Twells R, Orozco G, Brice A, Weber J, Heredero L, Scheufler K, Riley B, Allotey R, Nothers C, Hillermann R, Lunkes A, Khati C, Stevanin G, Hernandez A, Magariño C, Klockgether T, Durr A, Chneiweiss H, Enczmann J, Farrall M, Beckmann J, Mullan M, Wernet P, Agid Y, Freund H-J, Williamson R, Auburger G, Chamberlain S (1993): Chromosomal assignment of the second locus for autosomal dominant cerebellar ataxia (SCA2) to chromosome 12q23-24.1. Nature Genet 4: 295-299

Lathrop GM, Lalouel JM, Julier C, Ott J (1984): Strategies for multilocus linkage analysis in humans. Proc Natl Acad Sci USA 81 : 3443-3446

Lima L. Coutinho P (1980): Clinical criteria for diagnosis of Machado-Joseph disease: report of a non-Azorean Portuguese family. Neurology 30: 319-322

NIH/CEPH collaborative mapping group (1992): A comprehensive genetic linkage map of the human genome. Science 258; 67-86

Orr HT, Chung M, Banfi S, Kwiatkowski TJ Jr, Servadio A, Beaudet AL, MaCall AE, Duvick LA, Ranum LPW, Zoghbi HY (1992): Expansion of an unstable trinucleotide CAG repeat in spinocerebellar ataxia type 1. Nature Genet 4: 221-226

Ott J (1974): Estimation of the recombination fraction in human pedigrees: efficient computation of the likelihood for human linkage studies. Am J Hum Genet 26: 588-594

Polymeropoulos MH, Rath DS, Xiao H, Merril CR (1990): Trinucleotide repeat polymorphism at the human pancreatic phospholipase A-2 gene (PLA2). Nucleic Acids Res 18: 7468

Pulst S-M, Nechiporuk A, Starkman S (1993): Anticipation in spinocerebellar ataxia type 2. Nature Genet 5: 8-10

Ranum LPW, Duvick LA, Rich SS, Schut LJ, Litt M, Orr HT (1991): Localization of the autosomal dominant HLA-linked spinocerebellar ataxia (SCA1) locus, in two kindreds, within an 8-cM subregion of chromosome 6p. Am J Hum Genet 49: 31-41

Sasaki H, Wakisaka A, Tashiro K, Hamada K, Shima K (1991): Clinical study of gene locus heterogeneity in hereditary olivopontocerebellar atrophy (OPCA)-Report of 2 pedigrees affected with non SCA1 type OPCA. Clin Neurol (Tokyo) 31: 1170-1176

Sasaki H, Wakisaka A, Tashiro K, Hamada T, Shima K (1992): Linkage study of hereditary olivopontocerebellar atrophy: Genetic evidence for locus heterogeneity in Japanese cases. Clin Neurol (Tokyo) 32: 17-22

Weissenbach J, Gyapay G, Dib C, Vignal A, Morissette J, Millasseau P, Vaysseix G, Lathrop M (1992): A second-generation linkage map of the human genome. Nature 359: 794-801

Zoghbi HY, Jodice C, Sandkuijl LA, Kwiatkowski TJ Jr, McCall AE, Huntoon SA, Lulli P, Spadaro M, Litt M, Cann HM, Frontali M, Terrenato L (1991): The gene for autosomal dominant spinocerebellar ataxia (SCA1) maps telomeric to the HLA complex and is closely linked to the D6S89 locus in three large kindreds. Am J Hum Genet 49: 23-30

Vol. 39, No. 3, 1994 